\title{
Polarization Engineering in Photonic Crystal Waveguides for Spin-Photon Entanglers
}

\author{
A. B. Young, ${ }^{1}$ A. C. T. Thijssen, ${ }^{2}$ D. M. Beggs,${ }^{2}$ P. Androvitsaneas,,${ }^{2}$ L. Kuipers,${ }^{3}$ J. G. Rarity, ${ }^{1}$ S. Hughes,${ }^{4}$ and R. Oulton ${ }^{2,1}$ \\ ${ }^{1}$ Department of Electrical and Electronic Engineering, University of Bristol, \\ Merchant Venturers Building, Woodland Road, Bristol BS8 1UB, United Kingdom \\ ${ }^{2}$ Centre for Quantum Photonics, H.H. Wills Physics Laboratory, University of Bristol, \\ Tyndall Avenue, Bristol BS8 1TL, United Kingdom \\ ${ }^{3}$ Center for Nanophotonics, FOM Institute AMOLF, Science Park 104, 1098 XG Amsterdam, Netherlands \\ ${ }^{4}$ Department of Physics, Queen's University, Ontario, Canada K7L $3 N 6$
}

(Received 18 June 2014; revised manuscript received 3 July 2015; published 6 October 2015)

\begin{abstract}
By performing a full analysis of the projected local density of states (LDOS) in a photonic crystal waveguide, we show that phase plays a crucial role in the symmetry of the light-matter interaction. By considering a quantum dot (QD) spin coupled to a photonic crystal waveguide (PCW) mode, we demonstrate that the light-matter interaction can be asymmetric, leading to unidirectional emission and a deterministic entangled photon source. Further we show that understanding the phase associated with both the LDOS and the QD spin is essential for a range of devices that can be realized with a QD in a PCW. We also show how suppression of quantum interference prevents dipole induced reflection in the waveguide, and highlight a fundamental breakdown of the semiclassical dipole approximation for describing lightmatter interactions in these spin dependent systems.
\end{abstract}

Nanophotonic structures are routinely used to enhance light-matter interactions by modifying the density of electromagnetic (EM) field modes. This is often simplified to a scalar quantity, the local density of states (LDOS). However we show that the EM field modes also contain important phase information, which interacts with a phasedependent emitter in a nontrivial, nonintuitive way. This extra phase information is vital in practical designs of integrated quantum photonic circuits, a leading contender for future quantum technologies [1].

In a quantum photonic circuit, information may be stored and transmitted via photons. Photons suffer little from decoherence, and single qubit gates are straightforward. Less straightforward is the ability to create two qubit gates as direct photon-photon interactions are extremely weak. Quantum dots (QDs) have the potential to mediate photonphoton interactions acting as an artificial atom. Its solidstate nature means that it is relatively simple to enhance the light-matter interaction by incorporating it into nanophotonic structures. Simultaneously the electron spin states in QDs have shown long coherence times ( $\mu \mathrm{s})[2,3]$, and ease of optical initialisation, coherent control, and readout have all been demonstrated $[2,4,5]$. Thus the potential exists to use the QD spin to mediate deterministic photon-photon interactions.

If future devices are to be part of an integrated quantum photonic chip then a promising platform is a photonic crystal waveguide (PCW) and cavities [6]. A QD embedded in a PCW has already been recognized as an excellent single photon source [7-10]. This is because PCWs are approximately "one dimensional," where most of the energy from the emitter couples to the waveguide.
Accordingly simple "one-dimensional-atom" models $[11,12]$ may be applied to a PCW. In this Letter, we consider the coupling between polarized spin-dependent transitions of a QD trion to a PCW. We demonstrate that there is a complex interplay between the polarization structure of the PCW mode, the QD spatial location, and its spin state, leading to different functionalities that are not predicted by a standard one-dimensional atom model.

A two-dimensional PC is formed from a slab of dielectric containing periodically spaced air holes which modulate the refractive index, giving rise to an in-plane photonic band gap. The resulting confinement dramatically reduces the LDOS, relative to bulk material, into which a dipole can emit [8]. By incorporating a line of missing holes a waveguide is formed [see Fig. 1(a)]. The propagation of light along the waveguide supports slow light modes [13], which increase the LDOS in the waveguide region. As a result, the dominant modes for dipole emission are into this region thus forming a one-dimensional "wirelike" waveguide structure [14]. In contrast, in a standard waveguide the bulk LDOS is not significantly modified, and light scattered from the emitter is mainly directed into leaky modes.

Another significant difference between a standard planar waveguide and a PCW is the polarization state of the light propagating inside the structure. A standard waveguide supports a TE mode which is constant along the length of the guide. However, the PCW supports bound Bloch modes (BMs) with components of both $E_{x}$ and $E_{y}$ fields, that vary across one lattice period. Hence different locations inside the PCW support different superpositions of $E_{x}$, and $E_{y}$ 

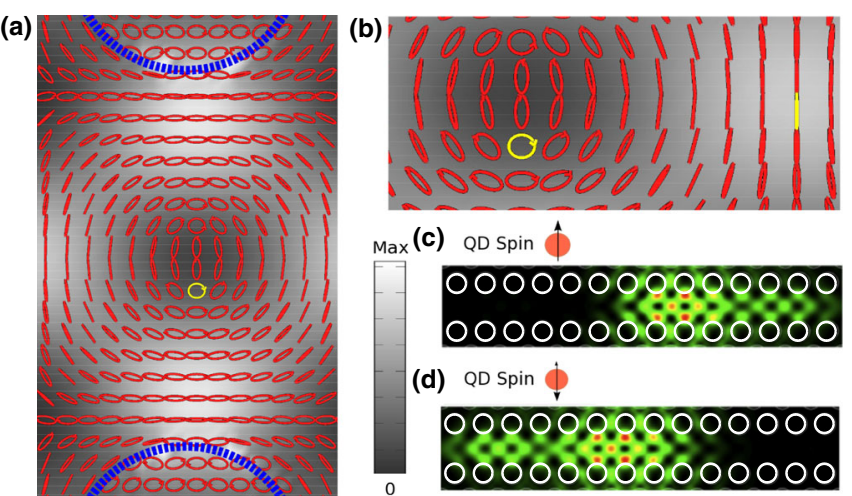

$\operatorname{Max}(c)$ QD Spin $\hat{\phi}$

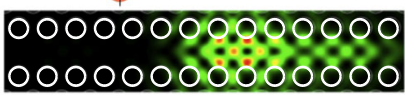

(d) QD Spin

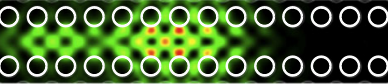

FIG. 1 (color online). (a) Zoom in of a $W 1 \mathrm{PCW}$ made from a suspended slab of GaAs with air holes (marked with dashed blue line), lattice constant $a=250 \mathrm{~nm}$ and the hole size is $0.34 a$. A line of holes is missing through the center forming the waveguide. Gray scale background shows field intensity, red markings show polarization ellipse, where straight lines represent linear polarization. (b) Zoom of specific area where the yellow circle represents the $C$ point and yellow line the $\mathbf{E}_{y}$ polarized point we consider in this Letter. FDTD simulations showing emission from a negatively charged QD at the identified $C$ point for (c) spin-up $\left(\sigma_{+}\right.$polarized $)$, and $(\mathrm{d})$ spin-down $\left(\sigma_{-}\right.$polarized $)$.

with a fixed relative phase that varies spatially. At each point the field may be expressed as a polarization ellipse, as shown in Fig. 1(a). There are clearly points where the ellipse becomes circular which corresponds to a " $C$-point" singularity $[15,16]$, and also where the ellipse collapses to a line ( $L$ line) where the polarization is linear. It is clear that the polarization of the mode is intricate, with an arbitrary point in the PCW $\left(\mathbf{r}_{0}\right)$ showing an arbitrary local electric field polarization, with $\mathbf{e}_{k}\left(\mathbf{r}_{0}\right)=\alpha \mathbf{E}_{x}+e^{i \phi} \beta \mathbf{E}_{y}$.

The QDs themselves are modeled as pointlike emitters. In addition, negatively doped QDs with a resident electron spin undergo strict selection rules that couple to $\sigma_{+}$circularly polarized light for spin-up $(|\uparrow\rangle)$ and $\sigma_{-}$ light for spin-down $(|\downarrow\rangle)$ [17]. The QD spin transitions may be modeled as superpositions of orthogonal dipoles aligned along $x$ and $y$, i.e., $\boldsymbol{\mu}=\alpha \boldsymbol{\mu}_{x}+e^{i \phi} \beta \boldsymbol{\mu}_{\boldsymbol{y}}$, where $\boldsymbol{\mu}$ represents a unit vector in the dipole direction. In bulk or simple dielectric structures, the coupling strength of the emitter is calculated to be proportional to the scalar product of $\left|\boldsymbol{\mu} \cdot \mathbf{E}\left(\mathbf{r}_{0}\right)\right| /|\boldsymbol{\mu}| \cdot\left|\mathbf{E}_{\max }\right|$, with the available LDOS proportional to $\left|\mathbf{E}_{\max }\right|^{2}$. However, the LDOS does not contain the full phase information present in the EM field modes. This necessitates a departure from this model and the use of a Green function analysis $[9,18,19]$, where the radiative coupling between the dipole and the waveguide mode is proportional to $\boldsymbol{\mu}^{\dagger} \cdot \mathbf{G}\left(r_{0}, r_{0}\right) \cdot \boldsymbol{\mu}$. The Green's function describes the response at $\mathbf{r}$ to an oscillating dipole at $\mathbf{r}_{0}$.

In the frequency domain, the Green's function for the waveguide mode is described through [9] ( $\omega$ is implicit)

$$
\begin{aligned}
\mathbf{G}_{w}\left(\mathbf{r}, \mathbf{r}_{0}\right)= & \mathbf{G}_{\mathrm{f}}\left(\mathbf{r}, \mathbf{r}_{0}\right)+\mathbf{G}_{\mathrm{b}}\left(\mathbf{r}, \mathbf{r}_{0}\right) \\
= & \frac{i a \omega}{2 v_{g}}\left[\Theta\left(x-x_{0}\right) \mathbf{e}_{k}(\mathbf{r}) \mathbf{e}_{k}^{*}\left(\mathbf{r}_{0}\right) e^{i k\left(x-x_{0}\right)}\right. \\
& \left.+\Theta\left(x_{0}-x\right) \mathbf{e}_{k}^{*}(\mathbf{r}) \mathbf{e}_{k}\left(\mathbf{r}_{0}\right) e^{-i k\left(x-x_{0}\right)}\right],
\end{aligned}
$$

where $a$ is the lattice constant, $v_{g}$ is the group velocity, $\Theta$ is the Heaviside step function, $x_{0}$ is the $x$ coordinate of the dipole, $\mathbf{e}_{k}(\mathbf{r})$ is the propagating mode for wave number $k$, normalized according to $\int_{V_{c}} \epsilon(\mathbf{r})\left|\mathbf{e}_{k}(\mathbf{r})\right|^{2} d \mathbf{r}=1$, where $V_{c}$ is the spatial volume of a PC unit-cell, with $\epsilon(\mathbf{r})$ the dielectric function. The first (second) term in Eq. (1) represents the Green's function for the forward (backward) propagating mode. An arbitrary point in the PCW $\left(\mathbf{r}_{0}\right)$ will thus have a local electric field polarization $\mathbf{e}_{k}\left(\mathbf{r}_{0}\right)=\alpha \mathbf{E}_{x}+e^{i \phi} \beta \mathbf{E}_{y}$, for light that is propagating in a forward propagating $\mathrm{BM}$. Whereas in the backwards propagating BM, $\mathbf{e}_{k}\left(\mathbf{r}_{0}\right)=\alpha \mathbf{E}_{x}+e^{-i \phi} \beta \mathbf{E}_{y}$. We now consider a specific point in the PCW where the field is circular ( $C$ point), i.e., where $\alpha=\beta$, and $\phi=\pi / 2$. Here we find if one sets $\boldsymbol{\mu}=\boldsymbol{\sigma}_{+}$then (excluding constants) $\boldsymbol{\mu}^{\dagger} \cdot \mathbf{G}_{\mathrm{f}}\left(\mathbf{r}_{0}, \mathbf{r}_{0}\right)$. $\boldsymbol{\mu}=1$ and $\boldsymbol{\mu}^{\dagger} \cdot \mathbf{G}_{\mathrm{b}}\left(\mathbf{r}_{0}, \mathbf{r}_{0}\right) \cdot \boldsymbol{\mu}=0$. Hence a right (left) circularly polarized dipole will only couple to the forward (backward) propagating mode.

This is confirmed by performing in-house FDTD simulations of a $W 1$ waveguide with slab thickness of $0.56 a$, hole radius of $0.34 a$, where $k a / 2 \pi=0.39$ and $v_{g}=c / 88$. In Fig. 1(c) we consider a $|\uparrow\rangle\left(\left|\sigma_{+}\right\rangle\right.$dipole) located at the $C$ point and in Fig. 1(d) a $|\downarrow\rangle\left(\left|\sigma_{-}\right\rangle\right.$dipole). Both show a unidirectional emission, dependent on spin orientation, in concurrence with the Green function analysis above. This striking result is due to the spin helicity in this system breaking the symmetry. Recent work has shown partial spin path correlations in other structures $[20,21]$. We show here, for the first time to our knowledge, how to precisely engineer these correlations, which is in excellent agreement with recent measurements using near field microscopy techniques [22]. Spin-path entanglement is a natural consequence of this analysis. A $|\uparrow\rangle$ dipole emits photons in the forward direction in the state $|\mathrm{f}\rangle$, while a $|\downarrow\rangle$ dipole emits photons in the backwards direction in state $|b\rangle$. An equal superposition of $|\uparrow\rangle+|\downarrow\rangle$ results in the output state:

$$
|\psi\rangle_{\text {out }}=|\uparrow\rangle|\mathrm{f}\rangle+|\downarrow\rangle|\mathrm{b}\rangle,
$$

an entangled state of photon path and spin orientation.

The efficiency of the source is given by the $\beta$ factor, defined as $\beta=\Gamma_{w} /\left(\Gamma_{w}+\Gamma_{0}\right)$, where $\Gamma_{0}$ represents radiative losses to modes above the light line; typically this latter contribution is much smaller than radiative decay to the waveguide mode, and is computed to be around $0.1 \Gamma^{\mathrm{hom}}$, where $\Gamma^{\text {hom }}$ represents the decay in the homogenous bulk material. The coupling rate to waveguide modes, $\Gamma_{w}$, depends on the coupling to the projected LDOS. The rate of emission can be split into two parts: the rate forward is 
given by $\Gamma_{w}^{\mathrm{f}}=2 d_{0}^{2} \boldsymbol{\mu}^{\dagger} \cdot \mathbf{G}_{\mathrm{f}}\left(\mathbf{r}_{0}, \mathbf{r}_{0}\right) \cdot \boldsymbol{\mu} / \hbar \epsilon_{0}$ and the rate backwards, $\Gamma_{w}^{\mathrm{b}}=2 d_{0}^{2} \boldsymbol{\mu}^{\dagger} \cdot \mathbf{G}_{\mathrm{b}}\left(\mathbf{r}_{0}, \mathbf{r}_{0}\right) \cdot \boldsymbol{\mu} / \hbar \epsilon_{0}$, where $d_{0}$ is the dipole moment of the optical transition. At a $C$ point, a dipole aligned to the field for the forward propagating BM, will be orthogonal to the field of the backwards propagating BM. Hence we find the following rate for spontaneous emission at a $C$ point:

$$
\Gamma_{w}^{C}=\Gamma_{w}^{\mathrm{f}}=\frac{d_{0}^{2} \mathrm{e}_{0}^{2} a \omega}{2 v_{g} \epsilon_{0} \hbar}=\frac{d_{0}^{2} \eta\left(\mathbf{r}_{0}, \boldsymbol{\mu}\right) Q_{w}}{\epsilon_{0} \hbar V_{\mathrm{eff}} \epsilon_{s}},
$$

where we have introduced an effective mode volume for the waveguide mode, $V_{\text {eff }} \equiv 1 /\left(\epsilon_{s}\left|\mathbf{e}_{k}\left(\mathbf{r}_{0}\right)\right|^{2}\right)$, where the BM is at the antinode position, and $\epsilon_{s}$ is the slab dielectric constant in which the QD is embedded. The waveguide mode decay rate is defined as $\kappa_{w}=2 v_{g} / a$, so $Q_{w}=\omega / \kappa_{w}$. We have also introduced $\eta$, a spatial and polarization dependent function, varying between 0 and 1 , to account for deviations from the antinode and polarization coupling with the target PCW mode. In contrast, at a point where the polarization is linear, and if the dipole is aligned to the field, $\Gamma_{w}^{L}=\Gamma_{w}^{\mathrm{f}}+\Gamma_{w}^{\mathrm{b}}=2 \Gamma_{w}^{C}$. So despite the fact the dipole is aligned to the local field in both cases, the decay rate at the $C$ point is inherently half (assuming maximum coupling) of that at a point of linear polarization. This is due to the lifting of the polarization degeneracy between the forward and backward propagating modes. As such the density of available EM modes at a $C$ point is halved relative to a linear point where the local field contains no phase information. Using the PCW in Fig. 1, and assuming a dipole moment of $d_{0}=30 \mathrm{D}$ we find a rate of emission for a spin-photon entangled source at a $C$ point of $\Gamma_{w} \sim 1.7 \mathrm{GHz}$, corresponding to a Purcell factor of $P_{f}=\Gamma_{w} / \Gamma^{\text {hom }}=1.8$. This yields a beta factor of $\beta \sim 0.95$.

By allowing the spin to emit several photons in a row, large entangled photon states may easily be built up, useful for quantum metrology or one way quantum computation using the cluster state model $[23,24]$. The device may therefore operate as a pumped source (optically out of plane, or electrically) of spin-path entangled photons when the QD spin is located at the $C$ point, the only place in the waveguide where this is possible, due to the perfect correlation of spin with path. Such device operation could never be predicted using a simple linear-dipole and LDOS approach commonly employed in cavity QED.

As well as deterministic entangled photon sources, deterministic quantum gates would be a crucial component for scalable quantum devices. We now explore implications of considering polarization in PCWs when designing quantum circuits. To perform a general analysis of the propagation and scattering of light in the PCW we again take a Green function approach, where the total field in the PCW, including the QD, and homogenous input field $\mathbf{E}^{h}(\mathbf{r})$ may be expressed as $\mathbf{E}(\mathbf{r})=\mathbf{E}^{h}(\mathbf{r})+\mathbf{G}\left(\mathbf{r}, \mathbf{r}_{0}\right) \cdot \boldsymbol{\alpha} \cdot \mathbf{E}^{h}\left(\mathbf{r}_{0}\right)$, where $\quad \boldsymbol{\alpha}=\left\{\left(\alpha_{0} \boldsymbol{\mu} \boldsymbol{\mu}^{\dagger}\right) /\left[1-\alpha_{0} \boldsymbol{\mu}^{\dagger} \cdot \mathbf{G}\left(\mathbf{r}_{0}, \mathbf{r}_{0}\right) \cdot \boldsymbol{\mu}\right]\right\} \quad$ is the
QD polarizability, which includes coupling to the medium (while allowing for complex dipoles in a Cartesian coordinate system), and the bare polarizability $\alpha_{0}=\left(2 \omega_{0} d_{0}^{2} / \epsilon_{0} \hbar\right) /\left(\omega_{0}^{2}-\omega^{2}\right)$, where we have neglected nonradiative losses.

Now consider a photon injected in the waveguide mode from the left (homogeneous solution), $\mathbf{E}^{h}(\mathbf{r})=$ $\sqrt{(a / L)} \mathbf{e}_{k_{h}}(\mathbf{r}) e^{i k_{h} x}$. For a sufficiently long waveguide, the transmitted and reflected fields are given by $\mathbf{E}_{t}(\mathbf{r} ; x \rightarrow \infty)=\sqrt{(a / L)} \mathbf{e}_{k_{h}}(\mathbf{r}) e^{i k_{h} x}+\mathbf{G}_{w}\left(\mathbf{r} ; x \rightarrow \infty, \mathbf{r}_{0}\right) \cdot \boldsymbol{\alpha}$. $\sqrt{(a / L)} \mathbf{e}_{k_{h}}\left(\mathbf{r}_{0}\right) e^{i k_{h} x_{0}}$, and $\mathbf{E}_{\mathbf{r}}(\mathbf{r}, x \rightarrow-\infty)=\mathbf{G}_{w}(\mathbf{r} ; x \rightarrow$ $\left.-\infty, \mathbf{r}_{0}\right) \cdot \boldsymbol{\alpha} \cdot \sqrt{(a / L)} \mathbf{e}_{k_{h}}\left(\mathbf{r}_{0}\right) e^{i k_{h} x_{0}}$, where we assume $x_{0}=0$. The transmitted and reflected amplitudes are, respectively, given by $t(\omega)=\mathbf{E}_{t}(\mathbf{r} ; x \rightarrow \infty) / \mathbf{E}^{h}(\mathbf{r} ; x \rightarrow \infty)$ and $r(\omega)=\mathbf{E}_{\mathrm{r}}(\mathbf{r} ; x \rightarrow-\infty) / \mathbf{E}^{h}(\mathbf{r} ; x \rightarrow-\infty)$, which are derived to be

$$
t(\omega)=1+\frac{i \omega_{0} 2 \Gamma_{w}^{\mathrm{f}}}{\omega_{0}^{2}-\omega^{2}-i \omega_{0}\left(\Gamma_{w}^{\mathrm{f}}+\Gamma_{w}^{\mathrm{b}}+\Gamma_{0}\right)},
$$

and

$$
r(\omega)=\frac{i \omega_{0} 2 \Gamma_{w}^{\mathrm{f} \rightarrow \mathrm{b}} e^{2 i k_{h} x_{0}}}{\omega_{0}^{2}-\omega^{2}-i \omega_{0}\left(\Gamma_{w}^{\mathrm{f}}+\Gamma_{w}^{\mathrm{b}}+\Gamma_{0}\right)},
$$

where $\Gamma_{w}^{\mathrm{f} \rightarrow \mathrm{b}}$ is the scattering rate backwards given a forward injected BM.

Now considering the case of a linearly polarized dipole, on an $L$ line in the PCW with the same linear polarization [yellow line in Fig. 1(b)]. A photon with a narrow bandwidth relative to the dipole transition (weak excitation approximation) input into the forward propagating waveguide mode leads to the frequency dependent response in Fig. 2(a). On resonance $\left(\omega=\omega_{0}\right)$, the dipole will scatter with the rates $\Gamma_{w}^{\mathrm{f}}=\Gamma_{w}^{\mathrm{b}}=\Gamma_{w}^{\mathrm{f} \rightarrow \mathrm{b}}$. Hence $|t(\omega)|^{2} \approx 0$, and $|r(\omega)|^{2} \approx 1$, and scattering from a QD leads to reflection back along the waveguide as predicted in earlier works [25]. One observes a dipole-induced reflection [26] identical to that in a cavity-waveguide architecture [11,27]. The dipole induced reflection feature in Fig. 2(a) has a width of $\sim 14 \mathrm{GHz}$ based on the waveguide simulated in Fig. 1 again assuming $d_{0}=30 \mathrm{D}$. This compares favorably with drop filter cavity designs [11], where the transparency window has a width of $\sim 100 \mathrm{GHz}$. Optimizations away from the standard $W 1$ waveguide should result in the transparency window becoming even wider. We now consider a charged QD at this $L$ line; by initializing in the spin-up state $|\uparrow\rangle$, a resonant photon injected into the forward propagating mode after scattering will end up in the entangled state:

$$
|\psi\rangle=|b\rangle|+\rangle+|f\rangle|-\rangle,
$$

where $|+\rangle=|\uparrow\rangle+|\downarrow\rangle$, and $|-\rangle=|\uparrow\rangle-|\downarrow\rangle$. Also, since along $L$ lines the local field has no fixed phase relation 

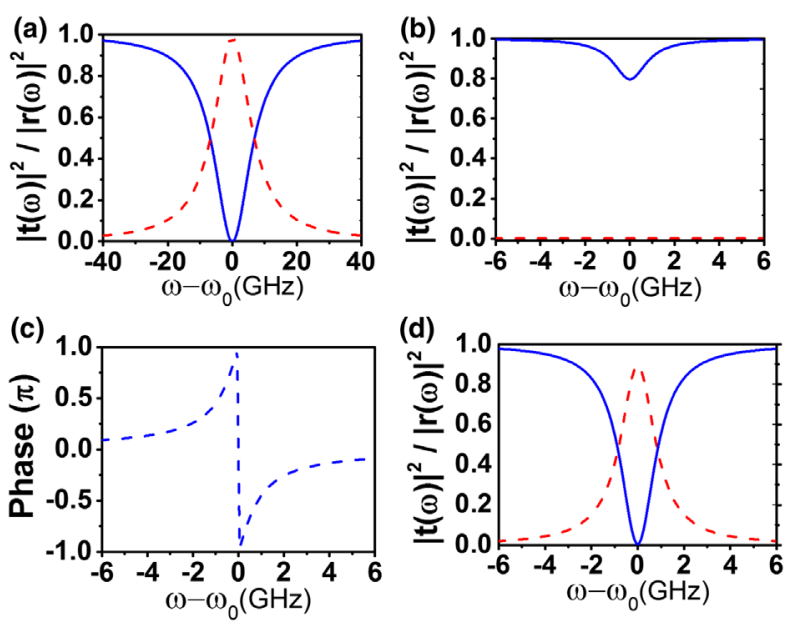

FIG. 2 (color online). Transmitted (blue line) and reflected (dashed red line) intensity as a function of detuning for (a) the linear $E_{y}$ dipole placed at a point in the PCW with pure $E_{y}$ polarized light, (b) the $\sigma_{+}$dipole at a $\sigma_{+}$polarized $C$ point, with (c) the accompanying phase shift on the transmitted signal as a function of detuning. (d) An $E_{y}$ dipole at a $\sigma_{+}$polarized $C$ point. All plots use the $W 1$ waveguide shown in Fig. 1, with parameters $\Gamma_{0}=0.1 \Gamma^{\mathrm{hom}}$ and $d_{0}=30 \mathrm{D}$.

between $E_{x}$ and $E_{y}$, the local field at the QD location $\left(\mathbf{r}_{0}\right)$ is the same in both forward and backward propagating directions, i.e., $\mathbf{e}_{k}\left(\mathbf{r}_{0}\right)=\mathbf{e}_{k}^{*}\left(\mathbf{r}_{0}\right)$. This allows one to encode photons via their path $(|f\rangle$ or $|b\rangle)$ and realize a fully deterministic spin-photon interface [28-30].

At a point where the local polarization is circular one sees a significant departure from the above. Figure 2(b) is a plot of the frequency dependant response to a forward propagating photon for a right circularly polarized dipole at a $C$ point [yellow circle in Fig. 1(b)]. Since we inject photons into the forward propagating mode the field created at the dipole location $\left(\mathbf{r}_{0}\right)$ is $\sigma_{+}$polarized. For the case when the dipole is also $\sigma_{+}$polarized then we find that $\Gamma_{w}^{\mathrm{b}}=\Gamma_{w}^{\mathrm{f} \rightarrow \mathrm{b}}=0$, on resonance and $\Gamma_{0}=0.1 \Gamma^{\mathrm{hom}}$, then $|r(\omega)|^{2} \approx 0$ and $|t(\omega)|^{2} \approx 0.8$. In this instance no light is reflected but is transmitted with a $\pi$ phase shift due to the interaction with the dipole. The reduction in the transmitted intensity is due to out of plane scattering. Since the $C$ point considered here is not at a field antinode, we find $\eta\left(\mathbf{r}_{0}, \boldsymbol{\mu}\right) \sim 0.25$. Optimizing the PCW structure to increase $\eta\left(\mathbf{r}_{0}, \boldsymbol{\mu}\right)$ will increase $\Gamma_{w}^{\mathrm{f}}$, improving the $\beta$ factor to give near unit transmission with a $\pi$ phase shift. If the dipole is $\sigma_{-}$polarized, then $\Gamma_{w}^{\mathrm{f}}=\Gamma_{w}^{\mathrm{f} \rightarrow \mathrm{b}}=0$; i.e., there is no interaction and the photon transmits without a phase shift.

Considering a two level system model, if the dipole is linearly polarized (interacting equally with $\sigma_{+}$and $\sigma_{-}$), then $\Gamma_{w}^{\mathrm{f}}=\Gamma_{w}^{\mathrm{b}}=\Gamma_{w}^{\mathrm{f} \rightarrow \mathrm{b}}$, and at the dipole resonance $|t(\omega)|^{2} \approx 0,|r(\omega)|^{2} \approx 0.9$ as in Fig. 2(d). Near unit reflection and a zero in transmission is caused by destructive interference between the scattered $\left(\sigma_{+}\right)$and the noninteracting $\left(\sigma_{-}\right)$components in the forward propagating direction. This is exactly the same as in Fig. 2(a) except the bandwidth and intensity of the dipole induced reflection feature is reduced. This is due to polarization mismatch and because the $C$ point is moved from the antinode of the BM, giving $\eta\left(\mathbf{r}_{0}, \mathbf{n}_{R}\right) \sim 0.125$.

In contrast for a charged QD at the $C$ point, if the spin is $|\downarrow\rangle$, then there is no interaction and a forward injected resonant photon will transmit. If the spin is $|\uparrow\rangle$, then the light transmits with a $\pi$ phase shift. If we prepare the QD spin in an equal superposition $(|+\rangle)$, then after interaction with a forward injected resonant photon we have the state

$$
|\psi\rangle_{\text {out }}=-|f\rangle|\uparrow\rangle+|f\rangle|\downarrow\rangle=-|f\rangle|-\rangle,
$$

where we have set $\Gamma_{0}=0$ for simplicity. This output state clearly does not correspond with the semiclassical result for a simple two level system in Fig. 2(d), there is no longer an available backwards propagating photon state. It is clear from this equation that the addition of spin into the system prevents destructive interference in the forward propagating direction. Measurement of a transmitted photon rotates the spin from the state $|+\rangle \rightarrow|-\rangle$. However if one chooses to measure the phase of the forward propagating photon (e.g., with a Mach-Zehnder interferometer) then spin-path entanglement is a natural consequence [31]. This predicts a stark contrast between a charged QD at a $C$ point, where one always sees transmission, and a fine-structure split neutral QD where one always sees a reflection. It further contrasts with the incoherent spontaneous emission result in Eq. (2) where one would detect output photons in the forward and backward mode with equal probability. This result highlights the role that coherence and quantum entanglement can play in spin mediated light-matter interactions, emphasising the care that one needs to take when making predictions about light propagation in nanophotonic structures. It is key to have a full description of the field of the local photonic environment, and also the nature of the dipole emitter to which it couples.

In conclusion we demonstrate, using a rigorous Green function method, that the LDOS in complex nanophotonic structures such as PCWs has important phase information that must not be neglected. We show the importance of this by considering a QD spin emitter in a PCW, and show that one may control the direction of photon emission by controlling the spin orientation. Entangled photon sources may be generated at a $C$-point polarization singularity while at both $C$ points and $L$ lines one may entangle photons via dipole induced reflection, all with $>90 \%$ efficiency. Most importantly, we develop a general mathematical framework to understand the interaction between dipoles and fields in chiral photonic structures, and show the limitations of a semiclassical analysis, where suppression of quantum interference prevents the dipole induced reflection of photons. 
The authors acknowledge helpful discussions with P. Lodahl, B. Lang, and R. Ge. EM simulations were carried out using the computational facilities of the Advanced Computing Research Centre, University of Bristol. This work has been funded by Future Emerging Technologies (FET)-Open FP7-284743 [project Spin Photon Angular Momentum Transfer for Quantum Enabled Technologies (SPANGL4Q)]. R. O. was sponsored by Engineering and Physical Sciences Research Council (EPSRC) Grant No. EP/G004366/1, and J.G.R. is sponsored under European Research Council (ERC) Grant No. 247462 Quantum Optics in Wavelength Scale Structures (QUOWSS). This work is part of the research program of the Stichting voor Fundamenteel Onderzoek der Materie, which is financially supported by the Nederlandse Organisatie voor Wetenschappelijk Onderzoek. D. M. B. acknowledges support from a Marie Curie individual fellowship, and S.H. acknowledges funding from the Natural Sciences and Engineering Research Council of Canada.

Note added.-Recently, we became aware of two related works: Ref. [31] considers a CNOT gate implementation in similar structures, and Ref. [32] shows directionality of emission from single atoms coupled to optical fiber.

[1] A. Politi, M. J. Cryan, J. G. Rarity, S. Yu, and J. L. O'Brien, Science 320, 646 (2008).

[2] K. De Greve et al., Nat. Phys. 7, 872 (2011).

[3] A. Greilich, D. R. Yakovlev, A. Shabaev, A. L. Efros, I. A. Yugova, R. Oulton, V. Stavarache, D. Reuter, A. Wieck, and M. Bayer, Science 313, 341 (2006).

[4] S. G. Carter, T. M. Sweeney, M. Kim, C. S. Kim, D. Solenov, S. E. Economou, T. L. Reinecke, L. Yang, A. S. Bracker, and D. Gammon, Nat. Photonics 7, 329 (2013).

[5] D. Press, K. De Greve, P. L. McMahon, T. D. Ladd, B. Friess, C. Schneider, M. Kamp, S. Hofling, A. Forchel, and Y. Yamamoto, Nat. Photonics 4, 367 (2010).

[6] J. L. O'Brien, A. Furusawa, and J. Vuckovic, Nat. Photonics 3, 687 (2009).

[7] V. S. C. Manga Rao and S. Hughes, Phys. Rev. B 75, 205437 (2007).

[8] G. Lecamp, P. Lalanne, and J. P. Hugonin, Phys. Rev. Lett. 99, 023902 (2007).
[9] P. Yao, V. S. C. Manga Rao, and S. Hughes, Laser Photonics Rev. 4, 499 (2010).

[10] T. Lund-Hansen, S. Stobbe, B. Julsgaard, H. Thyrrestrup, T. Sünner, M. Kamp, A. Forchel, and P. Lodahl, Phys. Rev. Lett. 101, 113903 (2008).

[11] E. Waks and J. Vuckovic, Phys. Rev. Lett. 96, 153601 (2006).

[12] A. Auffeves-Garnier, C. Simon, J.-M. Gerard, and J.-P. Poizat, Phys. Rev. A 75, 053823 (2007).

[13] T. Baba, Nat. Photonics 2, 465 (2008).

[14] D. Kleppner, Phys. Rev. Lett. 47, 233 (1981).

[15] M. Burresi, R. J. P. Engelen, A. Opheij, D. van Oosten, D. Mori, T. Baba, and L. Kuipers, Phys. Rev. Lett. 102, 033902 (2009).

[16] J. F. Nye, Proc. R. Soc. A 389, 279 (1983).

[17] See Supplemental Material at http://link.aps.org/ supplemental/10.1103/PhysRevLett.115.153901 for Quantum dot energy level diagrams.

[18] S. Hughes, Opt. Lett. 29, 2659 (2004).

[19] M. Wubs, L. G. Suttorp, and A. Lagendijk, Phys. Rev. A 70, 053823 (2004).

[20] F. J. Rodríguez-Fortuño, G. Marino, P. Ginzburg, D. O'Connor, A. Martínez, G. A. Wurtz, and A. V. Zayats, Science 340, 328 (2013).

[21] I. J. Luxmoore, N. A. Wasley, A. J. Ramsay, A. C. T. Thijssen, R. Oulton, M. Hugues, S. Kasture, V. G. Achanta, A. M. Fox, and M. S. Skolnick, Phys. Rev. Lett. 110, 037402 (2013).

[22] B. le Feber and N. Rotenberg, and L. Kuipers, Nat. Commun. 6, 6695 (2015).

[23] N. H. Lindner and T. Rudolph, Phys. Rev. Lett. 103, 113602 (2009).

[24] R. Raussendorf and H. J. Briegel, Phys. Rev. Lett. 86, 5188 (2001).

[25] J. T. Shen and S. Fan, Opt. Lett. 30, 2001 (2005).

[26] P. Kochan and H. J. Carmichael, Phys. Rev. A 50, 1700 (1994).

[27] S. Hughes and H. Kamada, Phys. Rev. B 70, 195313 (2004).

[28] L. M. Duan and H. J. Kimble, Phys. Rev. Lett. 92, 127902 (2004).

[29] C. Bonato, F. Haupt, S. S. R. Oemrawsingh, J. Gudat, D. Ding, M. P. van Exter, and D. Bouwmeester, Phys. Rev. Lett. 104, 160503 (2010).

[30] C. Y. Hu, A. Young, J. L. O'Brien, W. J. Munro, and J. G. Rarity, Phys. Rev. B 78, 085307 (2008).

[31] I. Sollner et al., Nat. Nanotechnol. 10, 775 (2015).

[32] R. Mitsch, C. Sayrin, B. Albrecht, P. Schneeweiss, and A. Rauschenbeutel, Nat. Commun. 5, 5713 (2014). 